\title{
Radiofrequency ablation therapy - the grave for GAVE (gastric antral vascular ectasia)?
}

Authors

Institution
Shajan Peter, C. Mel Wilcox

Division of Gastroenterology and Hepatology, Department of Medicine, University of Alabama at Birmingham, AL, USA submitted

30. January 2015

accepted after revision

20. February 2015

\section{Bibliography}

DOI http://dx.doi.org/

10.1055/s-0034-1391854

Published online: 13.4.2015

Endoscopy International Open

2015; 03: E128-E129

(c) Georg Thieme Verlag KG

Stuttgart - New York

E-ISSN 2196-9736

\section{Corresponding author}

Shajan Peter, MD

Department of

Gastroenterology

Basil Hirschowitz Endoscopic

Center of Endoscopic Excellence

6th Floor Jefferson Tower

625 19th Street South

Birmingham

AL 35249

USA

Fax: +1-205-934-8493

shajan@uab.edu
Gastric antral vascular ectasia (GAVE) is a distinct acquired entity presenting with small ectatic vessels localized to the gastric antrum and having characteristic endoscopic features. GAVE can be observed in patients with cirrhosis with and without portal hypertension and also in other conditions such as autoimmune disorders, scleroderma, chronic renal failure, cardiac disease, following bone marrow transplantation, and in patients with a left ventricular assist device [1-3]. The pathophysiology of GAVE makes it a separate entity distinct from portal hypertensive gastropathy. Alterations in systemic blood flow, the role of local acting vasoactive substances such as nitric oxide, gastrin, and prostaglandin, and mechanical stress resulting from altered gastric antral motility are some of the proposed mechanisms.

The prevalence of GAVE is unknown. Clinically, GAVE is often asymptomatic and often observed as an incidental finding at endoscopy performed for a variety of indications. Gastrointestinal bleeding is the most well recognized complication of GAVE, typically manifested as chronic iron deficiency anemia resulting in long-term transfusion dependence; acute overt bleeding is rare. Endoscopy remains an important modality not only in diagnosis, staging, and ability to obtain tissue for a definitive histological diagnosis, but also to determine reasonable therapeutic options. However, it is critical to recognize this disorder and not label the lesions as "gastritis". Ablative techniques leading to eradication of ectatic blood vessels remain the mainstay for endoscopic treatment and have included Nd:YAG laser, heater probe, cryotherapy, and argon plasma coagulation (APC). APC is the commonest technique used owing to its ease of use, it is less costly, and it has an excellent safety profile [4]. Recently, radiofrequency ablation (RFA) has been increasingly used as an alternative and especially in refractory cases of GAVE.
In this issue of the journal, Jana et al. present a clinical series of seven patients with refractory GAVE following APC who underwent RFA using the $\mathrm{HALO}^{90}$ system. They were successfully able to decrease transfusion dependence after an average of two sessions of RFA when followed for a mean of 6 months. The etiological spectrum of GAVE in this series included liver disease, autoimmune disorders such as systemic lupus erythematosus, scleroderma, and hypothyroidism. The study was significantly limited by the small number of patients, failure to obtain histology for diagnosis, short duration of follow-up, and lack of endoscopic resolution as an end point. The results, however, do parallel similar studies including a larger multicenter report from Europe of 24 patients which also showed a reduction in transfusion requirements after RFA [5-8].

The growing evidence points to RFA as feasible and safe in ablating GAVE lesions. The potential advantages of this technique are: (a) ability to deliver high energy coaptive coagulation of superficial mucosa including blood vessels, (b) wider surface area coverage of mucosa owing to the various electrodes sizes, e.g. HALO ${ }^{90}$ ULTRA $\left(5.2 \mathrm{~cm}^{2}\right)$ and $\operatorname{HALO}^{90}\left(2.6 \mathrm{~cm}^{2}\right)$ thereby avoiding skip areas, (c) contact technique with uniform zone of energy distribution and penetration such that deeper ectatic submucosal vascular channels are coagulated, and (d) technically easy to perform with a short learning curve. Nonetheless, there are several challenges with this technique, especially with regard to positioning and exact apposition to all parts of the antral mucosa owing to the fixed position of the electrode when mounted on the endoscope tip. This requires that the endoscope may have to be removed, the electrode rotated, and reintroduced multiple times. The newer through-the-scope internally rotatable ablating catheter may sidestep this disadvantage keeping in mind the smaller size of the electrode (1.2 $\mathrm{cm}^{2}$ ) [9] while delivering high pulses of up to 120 
per session. Also care should be taken not to clean the ablated mucosal tissue as this may lead to an increased risk of bleeding. What have we learned from these observations? First, not all GAVE lesions need to be treated unless they are symptomatic for blood loss. Second, the response to therapy will depend on the underlying microvascular anatomy and endoscopic appearance showing the extent and severity of lesions seen. Scanning electron microscopy images for GAVE lesions note the extensive tortuous dilatations across the surface often affecting deeper areas of the mucosal capillary network. Ectatic extension into deeper mucosa along with partial gastric rugal involvement and concomitant hypercontractility of the antrum might explain treatment failure and persistence of the lesions [10]. The endoscopic appearance has been classified as traditional watermelon type, diffuse or honeycomb variant, and recently proposed is a third variant with a nodular component but sharing the same histological characteristics [11]. It is not clear whether the unique microvascular architectural aspects of each type will play a role in the response to therapy, though it has been suggested that RFA may be advantageous in the diffuse and nodular type owing to larger surface area and depth of ablation provided. Likewise, band therapy has been offered as an alternative to ablation and a recent systematic review showed that more patients with GAVE treated by endoscopic band ligation were free from rebleeding (92\%) than those treated with APC (32\%) [4]. Clearly, more studies are needed to define which subset of GAVE will respond to either available therapies. Third, a clear distinction should be made between portal hypertensive gastropathy and GAVE in the presence of liver disease as both conditions can occur independently or coexist. The clinical presentation of chronic blood loss may be similar and endoscopically may be indistinguishable between the diffuse nodular portal hypertensive gastropathy and GAVE, though, therapies differ between them, the former responding to treatment of portal hypertension. Histology using the "GAVE score" comprising features such as fibrin thrombi and/or vascular ectasia, or spindle cell proliferation helps differentiating between them with good predictability and therefore may be necessary before initiation of treatment [12, 13].

The available data for RFA is limited owing to small study sizes, non-standardized definition of refractory GAVE and operator dependence. One might also argue whether clinical resolution of gastrointestinal bleeding, endoscopic ablation or histological resolution should be the primary end points of long-term management and follow-up.APC still has a beneficial role in the treatment of GAVE though its effectiveness varies upon individual expertise and should be offered as a first line therapy given its easy availability at most centers $[4,14,15]$. Larger prospective trials are needed to determine the ideal treatment option for GAVE. Ideally, randomized trials with head-to-head comparison between each endoscopic modality and long-term follow-up will be feasible in high volume centers or as multicentric studies. These will help in selecting the best cost effective modality, tailor exact dose of energy to be delivered, determine the ideal intervals required and predict risk factors for success or failure with the aim of complete eradication. Understanding the natural his- tory of GAVE played by roles of mechanical stress, humoral/immunological factors with hemodynamic alterations affecting the pathogenesis of associated co-morbid conditions and their histological correlation will help to target appropriate therapy, prevent recurrences, and avoid treatment resistance. The role of multimodal endoscopic therapy is a concept to explore understanding the complementary roles of each modality.

In conclusion, RFA is a safe and effective alternative endoscopic modality with a promising role in the treatment of symptomatic or refractory GAVE. Careful selection of patients who will benefit from this newer treatment will only be determined in larger prospective trials. This wealth of information will add RFA to the armamentarium of existing endoscopic therapies aiming to eradicate GAVE and potentially send it to eventually rest in peace in the grave.

\section{Competing interests: None}

\section{References}

1 Patwardhan VR, Cardenas A. Review article: the management of portal hypertensive gastropathy and gastric antral vascular ectasia in cirrhosis. Aliment Pharmacol Ther 2014; 40: 354-362

2 Fuccio L, Mussetto A, Laterza L et al. Diagnosis and management of gastric antral vascular ectasia. World J Gastrointest Endosc 2013; 5: 6-13

3 Alkurdi B, Monkemuller K, Khan AS et al. Gastric antral vascular ectasia: a rare manifestation for gastrointestinal bleeding in left ventricular assist device patients - an initial report. Dig Dis Sci 2014; 59: 2826 2830

4 Swanson E, Mahgoub A, MacDonald $R$ et al. Medical and endoscopic therapies for angiodysplasia and gastric antral vascular ectasia: a systematic review. Clin Gastroenterol Hepatol 2014; 12: 571 - 582

5 Dray X, Repici A, Gonzalez P et al. Radiofrequency ablation for the treatment of gastric antral vascular ectasia. Endoscopy 2014; 46: 963 -969

6 McGorisk T, Krishnan K, Keefer $L$ et al. Radiofrequency ablation for refractory gastric antral vascular ectasia (with video). Gastrointest Endosc 2013; 78: 584-588

7 Gross SA, Al-Haddad M, Gill KR et al. Endoscopic mucosal ablation for the treatment of gastric antral vascular ectasia with the HALO90 system: a pilot study. Gastrointest Endosc 2008; 67: 324-327

8 Raza N, Diehl DL. Radiofrequency ablation of treatment-refractory gastric antral vascular ectasia (GAVE). Surg Laparosc Endosc Percutan Tech 2015; 25: 79-82

9 Islam RS, Pasha SF, Fleischer DE. Refractory gastric antral vascular ectasia treated by a novel through-the-scope ablation catheter. Gastrointest Endosc 2014; 80: 896-897

10 Kern SE, Sitzmann JV. Microvascular architecture in a case of gastric antral vascular ectasia (watermelon stomach). Am J Gastroenterol 2014; 109: $449-451$

11 Marsteller WF, Lewin DN, Reuben A. The biopsy GAVE the diagnosis. Clin Gastroenterol Hepatol 2012; 10: e75-e76

12 Payen JL, Cales P, Voigt JJ et al. Severe portal hypertensive gastropathy and antral vascular ectasia are distinct entities in patients with cirrhosis. Gastroenterology 1995; 108: $138-144$

13 Qureshi K, Al-Osaimi AM. Approach to the management of portal hypertensive gastropathy and gastric antral vascular ectasia. Gastroenterol Clin North Am 2014; 43: 835-847

14 Boltin D, Gingold-Belfer R, Lichtenstein L et al. Long-term treatment outcome of patients with gastric vascular ectasia treated with argon plasma coagulation. Eur J Gastroenterol Hepatol 2014; 26: 588-593

15 Sebastian S, McLoughlin R, Qasim A et al. Endoscopic argon plasma coagulation for the treatment of gastric antral vascular ectasia (watermelon stomach): long-term results. Dig Liver Dis 2004; 36: 212 - 217 